\title{
Offspring of parents who were depressed were more likely to have a psychiatric disorder 10 years later
}

Weissman MM, Warner V, Wickramaratne P, et al. Offspring of depressed parents. 10 years later. Arch Gen Psychiatry 1997 Oct;54:932-40.

\section{Question}

Are the offspring of parents who are depressed more likely to have major depressive disorder (MDD) in adulthood compared with offspring of parents who are not depressed?

\section{Design}

Cohort study with follow up at 10 years.

\section{Setting}

New Haven, Connecticut, USA

\section{Participants}

The initial sample consisted of 220 offspring (age range $6-23$ y) from 91 families, including 153 offspring from 65 families in which $\geqslant 1$ parent met Research Diagnostic Criteria for a lifetime history of MDD and had received treatment at the Yale University Depression Research Unit; and 67 offspring from 26 families in which neither parent had a history of psychiatric illness (based on 4 interviews) who were identified from a community survey. Parents with and without depression were matched by group for age and sex. 182 offspring and 73 parents were interviewed at the 10 year follow up.

\section{Assessment of risk factors}

Parents with and without depression and spouses of parents were independently interviewed using the Schedule for Affective Disorders and Schizophrenia - Lifetime version, modified to include Research Diagnostic Criteria and Diagnostic and Statistical Manual of Mental Disorders 3rd edition, revised (DSM-III-R) criteria. An experienced clinician, blinded to diagnostic status of the offspring, assigned a DSM-III-R diagnosis to parents based on data from direct and informant interviews and from medical records.

\section{Main outcome measures}

DSM-III-R diagnosis of MDD and other psychiatric illnesses for each offspring was assigned by a blinded clinician using information from direct and informant interviews and medical records. Data on overall functioning (Global Assessment Scale), social adjustment (Social Adjustment Scale - Self-Report), and illness episodes were also collected.

\section{Main results}

Offspring of parents who were depressed had higher cumulative rates of MDD (relative risk [RR] 2.50, adjusted for age and sex, 95\% CI 1.38 to 4.54), phobias (adjusted RR 2.94, CI 1.03 to 8.41), panic disorder (2-tailed Fisher exact test $6.23, \mathrm{p}=0.004)$, and alcohol dependence (adjusted RR 4.93, CI 1.16 to 21.06) compared with offspring of parents who were not depressed. They received more outpatient treatment (adjusted odds ratio [OR] 2.11, CI 1.08 to 4.11 ) and had poorer overall functioning (adjusted OR 3.78, CI 1.50 to 9.56).

Offspring who had depressed parents and were depressed at the initial interview or 1 year later had poorer overall functioning (adjusted OR 4.34, CI 1.33 to 14.14), poorer social adjustment $(\mathrm{p}=0.01)$, reported more days on which they were depressed $(p=0.02)$, but were less likely to seek treatment when they felt it was needed (adjusted OR 12.85, CI 1.61 to 102.54) compared with offspring who had non-depressed parents and were depressed at the initial interview.

\section{Conclusion}

After 10 years, offspring of parents who were depressed had an increased risk of major depressive disorder, phobias, panic disorder, and alcohol dependence compared with offspring of parents who were not depressed.

Source of funding: in part, National Institute of Mental Health

For correspondence: Dr M M Weissman, College of Physicians and Surgeons of Columbia University, New York State Psychiatric Institute, 722 W 168th Street, New York, NY 10032 USA. Fax +1 212 5683534

\section{Commentary}

The study by Weissman et al has extended our knowledge about the risk of depression in offspring of depressed parents by following up for 10 years the children of parents with and without depression. The investigators acknowledge that their sample size is small and that the selection of offspring who had parents with a history of MDD from a treatment setting may have affected the seriousness of subsequent illnesses in the offspring. The results, however, not only support previous research but also provide more specific information about the time and rate of onset of mental health disorders in these offspring.

The results of this study are relevant to all nurses who assess patient health status. The US Agency for Health Care Policy and Research reported that $1 / 3$ to $1 / 2$ of patients with MDD are not diagnosed and that the total cost to society for untreated and undertreated depression is US\$16m annually. ${ }^{1}$ The effect of these statistics is augmented by the World Health Organisation prediction that mood disorders will be the number one public health problem of the 21 st century. To increase early diagnosis and treatment of depression therefore, it is imperative that nurses give special attention in assessment interviews to a family history of depression and be able to recognise the early symptoms of MDD.

The study findings are especially relevant to nurses who interact with mothers and their children. The authors suggest that children of depressed mothers are at high risk of MDD because of genetic factors and because of the negative effects of the mothers' depression on the parentchild relationship. Nurses can help by encouraging mothers' compliance with treatment and by being sensitive to early mental health problems in the children. They can provide age appropriate education to the children about their own risk of depression and about depression as an illness, with particular efforts to reduce their reluctance to seek treatment because of the perceived stigma associated with mental illness.

Lynda Slimmer, RN, PhD Professor of Nursing Elmhurst College Elmhurst, Illinois, USA

1 Agency for Health Care Policy and Research. Depression in primary care: volume 1. Detection and diagnosis. Clinical practice guideline. Rockville, MD: Agency for Health Care Policy and ville, MD: Agency
Research, 1993. 